\title{
Characterization of macrophages infiltrating peri-implantitis lesions
}

Tobias Fretwurst DDS ${ }^{1,3,5}$, Carlos Garaicoa-Pazmino DDS, $\mathrm{MS}^{2,5}$, Katja Nelson DDS, $\mathrm{PhD}^{3}$, William V. Giannobile DDS, MS, DMSc ${ }^{1}$, Cristiane H. Squarize DDS, MS, $\mathrm{PhD}^{1,5}$, Lena Larsson $\mathrm{PhD}^{1,4^{*}}$, Rogerio M. Castilho DDS, MS, $\mathrm{PhD}^{1,5^{*}}$

${ }^{I}$ Department of Periodontics and Oral Medicine, University of Michigan School of Dentistry, Ann Arbor, MI, USA.

${ }^{2}$ Department of Periodontology, Oregon Health \& Science University, School of Dentistry, Portland, OR, USA.

${ }^{3}$ Department of Oral- and Maxillofacial Surgery, Medical Center - University of Freiburg, Faculty of Medicine, University of Freiburg, Germany.

${ }^{4}$ Department of Periodontology, Institute of Odontology, University of Gothenburg, Gothenburg, Sweden.

${ }^{5}$ Laboratory of Epithelial Biology, Department of Periodontics and Oral Medicine, University of Michigan School, Ann Arbor, MI, USA.

Acknowledgments: TF was supported by a Research Scholarship of the Osteology Foundation.

Author contributions: RMC, CHS, WVG, LL, conceived the ideas; KN, TF, and CGP collected samples and data, TF performed experiments and analyzed data; and TF, LL, CGP, CHS, WVG, and RMC were involved in the writing.

Conflict of Interest and Source of Funding: The authors declared no conflicts of interest concerning the research, authorship, and/or publication of this article. The funders had no role in study design, data collection, and analysis, decision to publish, or preparation of the paper. The authors do not have any financial interests, either directly or indirectly, to the products or information identified in the paper.

\section{*Corresponding authors:}

Rogerio M. Castilho, DDS, MS, Ph.D.*

Laboratory of Epithelial Biology

Department of Periodontics and Oral Medicine

University of Michigan

1011 N University Ave, Room 2029C

Ann Arbor, MI, 48109-1078

Phone: (734) 647-2150

e-mail: rcastilh@umich.edu

This is the author manuscript accepted for publication and has undergone full peer review but has not been through the copyediting, typesetting, pagination and proofreading process, which may lead to differences between this version and the Version of Record. Please cite this article as doi: $10.1111 /$ CLR.13568

This article is protected by copyright. All rights reserved 
Lena Larsson, BSc, Ph.D.*

Department of Periodontology, Institute of Odontology, The Sahlgrenska Academy,

University of Gothenburg

Box 450, 40530 Gothenburg, Sweden

Phone: +46317863216

e-mail: lena.larsson@odontologi.gu.se

Running Title: Macrophage Polarization in Peri-implantitis

\title{
Author Contribution
}

Conceptualization T.F., L.L., C.G., K.N., W.V.G., and R.M.C.; Methodology, C.H.S.; R.M.C.; Investigation, T.F., L.L., C.G., and R.M.C.; Writing - Original Draft, T.F., L.L., C.G., and R.M.C.; Writing - Review \& Editing, C.G, C.H.S., R.M.C.; Funding acquisition, C.H.S., and R.M.C.; Resource C.H.S. and R.M.C.; Supervision, C.H.S., and R.M.C.

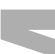

Abstract word count: 231 words

Total word count: 3,064 words

Total number of figures and tables: 1 table and 3 figures

Number of references: 35 references

\begin{abstract}
Objectives: The mechanisms involved in the initiation and progression of peri-implantitis lesions are poorly understood. It was the aim to determine the content and activation status of macrophages present in human peri-implantitis lesions and compare the current findings with the macrophage polarization associated with periodontitis lesions.
\end{abstract}

Material and Methods: A total of 14 patients were studied in this investigation. Seven were soft tissue biopsies from dental implants affected by peri-implantitis that required explantation. Seven biopsies were from chronic periodontal disease. Immunofluorescence stains were performed using biomarkers to identify macrophages $\left(\mathrm{CD}^{+} 8^{+}\right)$undergoing $\mathrm{M} 1$ polarization $\left(\mathrm{iNOS}^{+}\right)$and M2 polarization $\left(\mathrm{CD} 206^{+}\right.$), along with Hoechst 33342 to identify This article is protected by copyright. All rights reserved 
DNA content. All samples were stained and photographed, and double-positive cells for CD68 and iNOS, or CD68 and CD206 were quantified.

Results: All peri-implantitis biopsies examined revealed a mixed population of macrophages undergoing M1 and M2 polarization. Further analysis demonstrated the co-expression of iNOS and CD206, which indicates the presence of a heterogenic immune response on periimplantitis lesions. Macrophage polarization in peri-implantitis lesions presents a distinct pattern than in periodontitis. We observed a significant increase in the population of M1 macrophages on peri-implantitis samples compared to periodontal disease samples.

Conclusion: Our results demonstrate that peri-implantitis has higher numbers of macrophages displaying a distinct macrophage M1 polarization signature compared to periodontitis lesions. This pattern may explain, in part, the distinct nature of peri-implantitis progression versus periodontitis in humans.

MeSH term Keywords: Peri-implantitis; dental implants; inflammation; histology; macrophages; periodontitis; nitric oxide synthase

\section{Introduction}

Biological and technical complications can occur around osseointegrated dental implants leading to peri-implant diseases (Berglundh, Jepsen, Stadlinger, \& Terheyden, 2019; Derks \& Tomasi, 2015). Although the disease progression is a relatively well-known process, the etiology of peri-implantitis remains elusive.

Currently, studies on the inflammatory infiltrate surrounding peri-implantitis lesions have shown the predominant presence of plasma cells and lymphocytes (Berglundh, Zitzmann, \& Donati, 2011) along with neutrophils and macrophages (Berglundh et al., 2011; Carcuac \& Berglundh, 2014; Gualini \& Berglundh, 2003). Despite this composition assessment, there is limited information on the polarization status of macrophages in periimplantitis lesions. Macrophages undergo polarization in response to environmental cues. Notably, M1 macrophages are involved in the pro-inflammatory response that can be caused by bacteria and its cues, while M2 macrophages play a role in the resolution of inflammation and tissue repair (Palevski et al., 2017; Yu et al., 2016). Macrophages are also responsible for the production and secretion of cytokines. However, the secretory profile of macrophages undergoing M1 or M2 polarization differs. M1 macrophages express high levels of proinflammatory cytokines, especially IL-1ß and TNF- $\alpha$, reactive nitrogen, and oxygen 
intermediates; while M2 macrophages are typically associated with the production of IL-10, which is involved in the regulation of extracellular matrix, fibroblast function, and endothelial progenitor cells (King, Balaji, Le, Crombleholme, \& Keswani, 2014; Sica et al., 2000).

Aiming at an enhanced understanding of macrophage contribution to the pathogenic mechanisms of peri-implantitis, the current study characterized the polarization pattern of macrophages associated with peri-implantitis and compared these results with the macrophage composition of chronic periodontitis lesions.

\section{Materials and Methods}

The study was approved by the ethics committee of Charité Berlin, Germany (No EA4/050/13), the ethics committee of the University Medical Center Freiburg, Germany (No 268/13), and the University of Michigan Health Science Institutional Review Board (HUM00097548). This study was performed in accordance with the Helsinki Declaration of 1964, as revised in 2013.

\section{Study population}

Biopsies were harvested from tissues surrounding implants needing explantation due to peri-implantitis. Patients were enrolled consecutively at two study centers (the Department of Oral and Craniomaxillofacial Surgery at the University Medical Center Freiburg, and the Department of Oral and Maxillofacial Surgery at the Charite Campus Virchow Berlin) between August 2015 and December 2016. After evaluation and clinical indication for implant removal, patients were informed about the study conditions and signed written consent for the surgical procedure. Biopsy from periodontitis patients was previously reported (Garaicoa-Pazmino et al., 2019).

\section{Inclusion/exclusion criteria}

Patients with at least 1 implant diagnosed with peri-implantitis were enrolled in the study. The diagnosis of peri-implantitis was determined by clinical and radiographic parameters (presence of BOP and/or suppuration, changes in level of crestal bone with or without concomitant deepening PD) in accordance with 2017 World Workshop on the classification of periodontal and peri-implant diseases and conditions by the American Academy of Periodontology (AAP) and European Federation of Periodontology (EFP) (Berglundh et al., 2018). A diagnosis of Stage III or IV periodontitis was established on a 
tooth showing signs of loss of clinical attachment level (CAL) $\geq 5 \mathrm{~mm}$, probing depths $(\mathrm{PD}) \geq$ $4 \mathrm{~mm}$, bleeding on probing (BOP), and radiographic marginal bone loss of $\geq 33 \%$ (Caton et al., 2018; Papapanou et al., 2018).

Patients younger than 18 years of age, with previous surgical or periodontal therapy of the dental implant were excluded from the study. Immunocompromised patients, as well as patients undergoing radiation therapy or chemotherapy, were also excluded. Patients with periodontitis were also excluded if presenting with uncontrolled systemic disease or conditions known to alter bone metabolism (i.e., osteoporosis, osteopenia, hyperparathyroidism, or Paget's disease), pregnancy, history of oral cancer, sepsis or adverse outcomes to oral procedures, long-term use of antibiotics over 2 weeks in the past two months, smokers, former smokers, and patients taking medications known to modify bone metabolism. Inclusion criteria for periodontitis patients were previously reported (GaraicoaPazmino et al., 2019).

\section{Soft tissue harvesting procedure and/or processing}

A total of 7 peri-implant mucosa samples were obtained from 7 patients with dental implants diagnosed with peri-implantitis. The presence and polarization status of macrophages from peri-implantitis samples were compared with 7 soft tissue samples harvested from sites affected with periodontitis from 7 patients. Table 1 summarizes the demographic data from all included samples. Peri-implantitis tissue samples were obtained at the time of dental implant removal under local anesthesia, as described previously (Fretwurst et al., 2016). A circular incision with releasing incisions mesial and distal of the implant at a distance of $2 \mathrm{~mm}$ from the implant was performed in the soft tissue using a scalpel. A mucoperiosteal flap was mobilized, and the remaining inflamed peri-implant tissue was removed using a clamp and scalpel. The biopsies were placed in a $3.7 \%$ neutral buffered formalin solution (Otto Fischer GmbH, Saarbrücken, Germany) until further processing. The samples were embedded in Technovit 9100 (Heraeus Kulzer GmbH, Wehrheim, Germany) that is suitable for histological sections that may contain soft and hard tissues, following manufacturer's guidelines. Note that Technovit 9100 After complete embedding and cooling, sections were cut using either a rotary microtome with a glass diameter $400 \times 25 \times 100 \mathrm{~mm}$ (Leica, Nussloch, Germany) or a Leica microtome for paraffin-embedded tissues. Tissue sections of $5 \mu \mathrm{m}$ were prepared for histological examination. Periodontitis tissue samples were obtained at the University of Michigan School of Dentistry between September 2015 and March 2018 (Garaicoa-Pazmino et al., 2019). The periodontitis samples were obtained 
from the most accessible target lesions associated with traditional periodontal flap procedures. Open flap debridement and/or resective approaches were selected for periodontitis-affected patients. The biopsies were fixed in a $3.7 \%$ neutral buffered formalin solution, dehydrated and embedded in paraffin. The samples were stored as coded specimens not to reveal personal patient-related information.

\section{Immunofluorescence}

Antigen retrieval was performed using $10 \mathrm{mM}$ sodium citrate buffer ( $\mathrm{pH}$ 6). To block unspecific binding, the sections were incubated with 3\% (w/v) bovine serum albumin (BSA). After overnight incubation (at $\left.4^{\circ} \mathrm{C}\right)$ with primary antibodies (1:50 dilution) anti-CD68 (Rabbit Polyclonal Antibody, Proteintech, Rosemont, IL, USA), anti-iNOS (Mouse Monoclonal Antibody, R\&D Systems, Minneapolis, MN, USA), and anti-CD206 (Goat Polyclonal Antibody, R\&D Systems, Minneapolis, MN, USA); the sections were incubated with Alexa 488, Alexa 568 and Alexa 647-conjugated secondary antibodies (dilution 1:200) for $1 \mathrm{~h}$, and then stained with Hoechst 33342 (Sigma-Aldrich Corp., St. Louis, MO, USA) to visualize the individual nucleus of the cells. NIH ImageJ software (NIH, Bethesda, MD) was used to calculate the total number of cells per field presenting positive nuclear staining for Hoechst 33342. The DNA nuclear staining Hoechst 33342 is the staining of choice in immunofluorescence assays to determine the total number of live cells (cellular density) by providing high-quality resolution images. The omission of the primary antibody was used as negative controls. A QImaging ${ }^{\circledR}$ EXi Aqua ${ }^{\mathrm{TM}}$ monochrome digital camera (QImaging, Surrey, BC, Canada) attached to a Nikon Eclipse 80i microscope (Nikon, Melville, NY) was used to capture the region of interest (ROI) images from peri-implantitis and periodontitis samples. ROI was composed of connective tissue and associated inflammatory infiltrate, and the epithelial component of the gingival mucosa. All images were visualized with QCapturePro software (QImaging, Surrey, BC, Canada).

Identification and quantification of $\mathrm{iNOS}^{+} / \mathrm{CD} 8^{+}$(M1) and $C D 206^{+} / \mathrm{CD}^{+} 8^{+}$(M2) macrophage polarization

Tissue samples were photographed using a $20 \mathrm{X}$ objective, and 5 to 10 random independent fields were assessed using ImageJ software. Due to the number of channels limitation of the RGB color model, all samples were analyzed by combining CD68 (Alexa 568) staining with CD206 (Alexa 488) and Hoechst 33342 (Blue), or CD68 (Alexa 568) combined with iNOS (Alexa 647) and Hoechst 33342 (Blue) to identify and quantify triple- 
positive cells. As all generated images were in grayscale, and the specificity of each channel was given by the narrowband filters, the pseudocolor of the samples followed the RGB (red, green, and blue) channels. This strategy allowed consistent use of a split channel function for precise quantification of positive macrophages and the corresponding polarization. The $\mathrm{iNOS}^{+} / \mathrm{CD} 8^{+}$(M1-like macrophage) and CD206 ${ }^{+} / \mathrm{CD}^{+} 8^{+}$(M2-like macrophage) positive cells were analyzed and quantified using monochromatic images using the NIH ImageJ software (https://imagej.nih.gov/ij/) split-channel mode, followed by cell counting plugin (Schneider, Rasband, \& Eliceiri, 2012). The results were expressed as a percentage of positive double-stained cells among the total number of $\mathrm{CD} 68^{+}$cells. The analyses were done by three masked, independent examiners (LL, CGP, TF). The Kappa test was used to calculate the inter-examiner agreement, which indicates a moderate to a substantial agreement among examiners $(\mathrm{K}=0.63)$.

\section{Statistical Analysis}

Statistical analyses were performed using a one-way ANOVA or Student $t$-test to analyze two distinctive groups or sets of data. GraphPad Prism 8.00 (GraphPad Software, San Diego, CA) were used, and the asterisks denote statistical significance $\left({ }^{*} p<0.05\right.$; ${ }^{*} p<0.01$; $* * * p<0.001$; and NS $p>0.05)$.

\section{Results}

\section{Peri-implantitis lesions are populated with macrophages}

$\mathrm{CD} 8^{+}$cells (macrophages) were present in peri-implantitis, particularly within the inflamed soft tissue surrounding the implant (Fig. 1, red channel). From this group of cells, we decided to further identify the macrophages associated with an acute response phase characterized by M1 polarization (iNOS ${ }^{+}$cells) (Fig. 1A, cyan channel) and the macrophages associated with a resolution phase characterized by an M2 polarization $\left(\mathrm{CD} 206^{+}\right.$cells) (Fig. 1B, green channel). The cells were also stained with Hoechst 33342 nuclear stain. Merged images of CD68 and iNOS (Fig. 1A) and CD68 and CD206 (Fig. 1B) double-positive cells were present in all periimplantitis samples suggestive of a mix population of macrophages undergoing M1 and M2 polarization. Merged images containing all 4 channels (red: $\mathrm{CD}^{+} 8^{+}$, green: $\mathrm{CD}_{206}{ }^{+}$, cyan: $\mathrm{iNOS}^{+}$, and blue: Hoechst 33342) demonstrated the heterogeneity of macrophage polarization in peri-implantitis lesions (Fig. $1 \mathrm{C}$ and D). 
Our results showed that peri-implantitis samples presented a similar polarization pattern of macrophages, in which $53.74 \pm 5.98 \%$ of the macrophages are $\mathrm{iNOS}^{+} / \mathrm{CD}^{+} 8^{+}(\mathrm{M} 1$ polarization) and $56.34 \pm 6.91 \%$ are $C D 206^{+} / C D 68^{+}$(M2 polarization) (Fig. 2A). Also, we identified macrophages co-expressing iNOS and CD206 markers, indicating a heterogenic immune response during the process of peri-implantitis. When analyzing the distribution of macrophage polarization among all 7 patients, we observed that 1 patient presented macrophages with M1 polarization (i.e., samples 1); and 3 patients showed more macrophages with M2 polarization (i.e., samples 3, 4, 7) (Fig. 2B). Notably, we detected the presence of similar levels of M1 and M2 polarization in almost half of the samples (i.e., samples 2, 5, and 6) (Fig. 2B). Indeed, $\mathrm{CD}^{+}$cells (macrophages) comprised of $14.92 \pm 2.19 \%$ of the total number of cells found in each ROI (identified by Hoechst 33342) present in the connective tissue (cellular density), while only $7.35 \pm 1.44 \%$ were $\mathrm{iNOS}^{+} / \mathrm{CD}^{+} 8^{+}$ (M1 polarization) $(* * p<0.01)$, and $7.56 \pm 1.43 \%$ were $C D 206^{+} / C D 68^{+}$(M2 polarization) $(* * p<0.01)($ Fig. $2 \mathrm{C}$, Supplementary table 1).

\section{Peri-implantitis presents a distinct macrophage polarization pattern from periodontitis}

Here, we investigated the macrophages content within peri-implantitis lesions and compared it with our previously reported cohort of periodontitis patients (Garaicoa-Pazmino et al., 2019). Our results showed that soft tissues from periodontitis have an inflammatory infiltrate containing significant levels of CD68+ macrophages. Similar to the peri-implantitis samples, $\mathrm{CD} 8^{+}$cells in periodontitis presented either an M1 polarization detected by iNOS and/or an M2 polarization identified by $\mathrm{CD} 206^{+}$marker. When comparing the results from peri-implantitis cases and periodontitis, we found that peri-implantitis samples displayed higher numbers of $\mathrm{CD}^{+} 8^{+}$cells. An average of $14.92 \pm 2.19 \%$ of the cells present in the connective tissue of peri-implantitis were $\mathrm{CD}^{+} 8^{+}$cells, compare to $8.01 \pm 0.32 \% \mathrm{CD} 68^{+}$cells found in the periodontitis samples (Fig. $3 \mathrm{~A}, * * p<0.01$, Supplementary table 2). In addition, when comparing the subpopulations of M1 and M2 macrophage polarization, it was observed a statistically significant increase in the population of macrophage in M1 from peri-implantitis samples $(7.06 \pm 1.44 \%)$ compared to periodontal disease samples $(1.64 \pm 0.14 \%)$ (Fig. 3B, ** $p<0.01)$. In contrast, M2 macrophage polarization presented similar levels among periimplantitis (7.56 $\pm 1.44 \%$ ) and periodontitis (4.07 $\pm 0.20 \%$ ) samples (Fig. 3B, ns $p>0.05$, Supplementary table 3). Even after adjusting for the total number of macrophages (baseline corrected) from periodontitis and peri-implantitis, an increase in M1 polarization in periimplantitis samples still evident $(49.93 \pm 5.1 \%)$ compared with periodontitis $(21.04 \pm 2.3 \%)$ 
$(* * * p<0.001)$ (Fig. 3C). M2 polarization remained similar among both diseases, with $53.67 \pm 5.2 \%$ of the peri-implantitis macrophages and $47.64 \pm 2.3 \%$ of periodontitis samples presenting an M2 polarization (ns $p>0.05$ ) (Fig. 3C, Supplementary table 4).

\section{Discussion}

In the present study, we investigated the status of macrophage polarization in human peri-implantitis lesions. Interestingly, we found that peri-implantitis present a distinct signature of macrophage polarization as compared with periodontitis lesions.

Macrophage polarization has been a complex topic with conflicting data in periodontal disease research (Viniegra et al., 2018; Yang et al., 2017; Yu et al., 2016; S. Zhang et al., 2013). Much of the discrepancy may be associated with technical challenges, such as the selection of molecular markers capable of identifying changes in macrophage polarization. This is particularly seen during the selection of markers capable of identifying M1 polarization. There are fewer markers associated with M1 polarization when compared to the available markers for M2. iNOS was chosen as a functional marker for the M1 phenotype (Lisi et al., 2017). Antibodies capable of detecting inducible nitric oxide synthase (iNOS) provide specificity in the identification of macrophages presenting high levels of nitric oxide as part of a defense mechanism.

Moreover, the quantification of macrophages that are actively changing their polarization status as the disease progresses makes cellular quantification challenging (Parisi et al., 2018). Infection with Porphyromonas gingivalis resulted in an increase in M1 polarization of macrophages in an experimental model of periodontitis (Lam et al., 2016; Lam et al., 2014). Yet, little is known regarding the macrophage polarization pattern found in periimplantitis lesions. A study comparing the cellular composition in peri-implantitis lesions with periodontitis demonstrated a significant increase in macrophages in peri-implantitis compared to periodontitis lesion (Carcuac \& Berglundh, 2014). This data is in line with the results found in the present study, where a significant increase in total numbers of CD68+ macrophages was found in peri-implantitis tissues compared to periodontitis.

M1 macrophages have been detected in tissues around implants affected by periimplantitis (Fretwurst et al., 2016; Pettersson et al., 2017). The present study has demonstrated that, indeed, macrophages presenting M1 polarization are present in periimplantitis lesions, and M1 polarization is more predominant in peri-implantitis than in periodontitis, suggesting an intrinsic difference between the macrophage content and potential contribution to these diseases. The higher number of macrophages and the acute response 
depicted by the elevated M1 polarization observed in peri-implantitis lesions also suggest a robust response of the immune system against local factors; and, therefore, increased tissue destruction. Our histological data are consistent with clinical disease progression observed in peri-implantitis. Peri-implantitis has been characterized by acute inflammation, early disease onset, and a non-linear, accelerating pattern of bone loss when compared to periodontitis (Derks et al., 2016).

Similar to the observed in periodontal diseases, other inflammatory conditions are characterized by the infiltration of macrophage presenting a dynamic range of polarization. Current knowledge on macrophage polarization suggests that monocytes and macrophages exhibit high cellular plasticity depending on environmental cues and alternative controlling mechanisms. Such mechanisms can induce the classical (Th1) or alternative (Th2) activation of macrophages (Das et al., 2015; Mills \& Ley, 2014). Classical activation of macrophages is triggered by Th1-type cytokines like interferon-gamma or by lipopolysaccharides leading to the conversion of arginine and the production of NO by iNOS (Classen, Lloberas, \& Celada, 2009). Th2-type cytokines, including IL-4, IL-10, or IL-13, trigger the alternative activation of macrophages leading to the degradation of arginine and further repair of the damaged extracellular matrix. It is interesting to note that in wounds, the infiltration of macrophages is composed of classical and alternative macrophage polarization (Daley, Brancato, Thomay, Reichner, \& Albina, 2010), similar to the observed in our peri-implantitis and periodontitis samples. In fact, the complexity of macrophage polarization in periodontal disease supports the need for a new classification system for macrophage activation status other than M1 or M2. It has been proposed by Murray and colleagues a novel system that takes into account the source of the macrophages, the definition of the activators, and a common agreement on the markers for macrophages (Murray et al., 2014). Macrophages are also susceptible to constant transcriptional reprogramming. Xue and colleagues used the transcriptional activation states of macrophage in M1 versus M2 to identify nine distinct macrophage activation programs (Xue et al.,2014). Such findings could explain the double-positive staining of macrophages with iNOS and CD206, which were noted in the present and previous study by our group (Garaicoa-Pazmino et al., 2019).

Our results are exciting and align with previous publications that identified a substantial increase in the number of macrophages in peri-implantitis lesions compared to periodontitis (Carcuac \& Berglundh, 2014). We also demonstrate that peri-implantitis lesions are endowed with an increased population of M1 macrophages compared with periodontitis lesions suggesting an aggressive disease progression. The current limitation of this study is 
the small sample size. Small sample size has elevated chances to incorporate unforeseen bias, and the statistical results must be interpreted carefully. The current literature on macrophage polarization does not have a clear consensus on markers to identify M1 and M2 polarization, and emerging data suggest an increased complexity on the differentiation of macrophages dependent on environmental cues. Few studies proposed staining protocols for activated macrophages with iNOS (He et al., 2015; Tang, Zhao, Lei, Chen, \& Zhang, 2019), CCR7 (Wang, Li, Feng, Cheng, \& Li, 2019) for detecting M1 phenotypes, while others used CD206 (Viniegra et al., 2018) (Lee et al., 2018; Nawaz et al., 2017; C. Zhang et al., 2017) or CD163 (Ham et al., 2017; Wang et al., 2019) for M2 phenotypes. The presence of subtypes of macrophage polarization like M2a, M2b, M2c, and M2d also remains to be defined in periodontal diseases. Further studies are also needed to clarify whether macrophage polarization depends on implant material/surface treatment or local contributing factors as unique environmental determinants of macrophage differentiation in peri-implantitis lesions. The present data offer information for in-depth follow-up investigations and in-vitro investigations to elucidate a potential etiological pathway. Also, further clinical studies will help to shed light on the macrophage signature and their potential contribution to the transition of peri-implant mucositis into peri-implantitis.

In conclusion, our findings indicate that peri-implantitis patients co-express both M1 and M2 polarization within the same lesion. Additionally, peri-implantitis lesions display higher levels of macrophages dispersed in the soft tissues compared to periodontitis, and most interestingly, peri-implantitis samples also present an increase in the M1 polarization when compared to periodontitis samples. These results aid in a better understanding of the complex process of the pathogenesis of human peri-implantitis lesions.

\section{Acknowledgments}

The authors declared no conflicts of interest concerning the research, authorship, and/or publication of this article. TF was supported by the Osteology Foundation Research Scholars Program, Switzerland. The funders had no role in study design, data collection, and analysis, decision to publish, or preparation of the paper. The authors do not have any financial interests, either directly or indirectly, related to the products or information discussed in the paper.

\section{References}


Berglundh, T., Armitage, G., Araujo, M. G., Avila-Ortiz, G., Blanco, J., Camargo, P. M., . . . Zitzmann, N. (2018). Peri-implant diseases and conditions: Consensus report of workgroup 4 of the 2017 World Workshop on the Classification of Periodontal and Peri-Implant Diseases and Conditions. J Periodontol, 89 Suppl 1, S313-S318. doi:10.1002/JPER.17-0739

Berglundh, T., Jepsen, S., Stadlinger, B., \& Terheyden, H. (2019). Peri-implantitis and its prevention. Clin Oral Implants Res, 30(2), 150-155. doi:10.1111/clr.13401

Berglundh, T., Zitzmann, N. U., \& Donati, M. (2011). Are peri-implantitis lesions different from periodontitis lesions? J Clin Periodontol, 38 Suppl 11, 188-202. doi:10.1111/j.1600-051X.2010.01672.x

Carcuac, O., \& Berglundh, T. (2014). Composition of human peri-implantitis and periodontitis lesions. J Dent Res, 93(11), 1083-1088. doi:10.1177/0022034514551754

Classen, A., Lloberas, J., \& Celada, A. (2009). Macrophage activation: classical versus alternative. Methods Mol Biol, 531, 29-43. doi:10.1007/978-1-59745-396-7_3

Daley, J. M., Brancato, S. K., Thomay, A. A., Reichner, J. S., \& Albina, J. E. (2010). The phenotype of murine wound macrophages. J Leukoc Biol, 87(1), 59-67. doi:10.1189/jlb.0409236

Das, A., Sinha, M., Datta, S., Abas, M., Chaffee, S., Sen, C. K., \& Roy, S. (2015). Monocyte and macrophage plasticity in tissue repair and regeneration. Am J Pathol, 185(10), 25962606. doi:10.1016/j.ajpath.2015.06.001

Derks, J., Schaller, D., Hakansson, J., Wennstrom, J. L., Tomasi, C., \& Berglundh, T. (2016). Peri-implantitis - onset and pattern of progression. J Clin Periodontol, 43(4), 383-388. doi:10.1111/jcpe.12535

Derks, J., \&Tomasi, C. (2015). Peri-implant health and disease. A systematic review of current epidemiology. J Clin Periodontol, 42 Suppl 16, S158-171. doi:10.1111/jcpe.12334

Fretwurst, T., Buzanich, G., Nahles, S., Woelber, J. P., Riesemeier, H., \& Nelson, K. (2016). Metal elements in tissue with dental peri-implantitis: a pilot study. Clin Oral Implants Res, 27(9), 1178-1186. doi:10.1111/clr.12718 
Garaicoa-Pazmino, C., Fretwurst, T., Squarize, C. H., Berglundh, T., Giannobile, W. V., Larsson, L., \& Castilho, R. M. (2019). Characterization of macrophage polarization in periodontal disease. J Clin Periodontol, 46(8), 830-839. doi:10.1111/jcpe.13156

Gualini, F., \& Berglundh, T. (2003). Immunohistochemical characteristics of inflammatory lesions at implants. J Clin Periodontol, 30(1), 14-18.

Ham, J. S., Park, H. Y., Ryu, K. J., Ko, Y. H., Kim, W. S., \& Kim, S. J. (2017). Elevated serum interleukin-10 level and M2 macrophage infiltration are associated with poor survival in angioimmunoblastic T-cell lymphoma. Oncotarget, 8(44), 76231-76240. doi:10.18632/oncotarget.19301

He, D., Kou, X., Luo, Q., Yang, R., Liu, D., Wang, X., . . . Zhou, Y. (2015). Enhanced M1/M2 macrophage ratio promotes orthodontic root resorption. J Dent Res, 94(1), 129-139. doi:10.1177/0022034514553817

King, A., Balaji, S., Le, L. D., Crombleholme, T. M., \& Keswani, S. G. (2014). Regenerative Wound Healing: The Role of Interleukin-10. Adv Wound Care (New Rochelle), 3(4), 315-323. doi:10.1089/wound.2013.0461

Lam, R. S., O'Brien-Simpson, N. M., Holden, J. A., Lenzo, J. C., Fong, S. B., \& Reynolds, E. C. (2016). Unprimed, M1 and M2 Macrophages Differentially Interact with Porphyromonas gingivalis. PLoS One, 11(7), e0158629. doi:10.1371/journal.pone.0158629

Lam, R. S., O'Brien-Simpson, N. M., Lenzo, J. C., Holden, J. A., Brammar, G. C., Walsh, K. A., . . . Reynolds, E. C. (2014). Macrophage depletion abates Porphyromonas gingivalisinduced alveolar bone resorption in mice. J Immunol, 193(5), 2349-2362. doi:10.4049/jimmunol.1400853

Lee, S. H., Charmoy, M., Romano, A., Paun, A., Chaves, M. M., Cope, F. O., . . Sacks, D. L. (2018). Mannose receptor high, M2 dermal macrophages mediate nonhealing Leishmania major infection in a Th1 immune environment. J Exp Med, 215(1), 357375. doi:10.1084/jem.20171389

Lisi, L., Ciotti, G. M., Braun, D., Kalinin, S., Curro, D., Dello Russo, C., ... Navarra, P. (2017). Expression of iNOS, CD163 and ARG-1 taken as M1 and M2 markers of microglial polarization in human glioblastoma and the surrounding normal parenchyma. Neurosci Lett, 645, 106-112. doi:10.1016/j.neulet.2017.02.076 
Mills, C. D., \& Ley, K. (2014). M1 and M2 macrophages: the chicken and the egg of immunity. J Innate Immun, 6(6), 716-726. doi:10.1159/000364945

Murray, P. J., Allen, J. E., Biswas, S. K., Fisher, E. A., Gilroy, D. W., Goerdt, S., . . Wynn, T. A. (2014). Macrophage activation and polarization: nomenclature and experimental guidelines. Immunity, 41(1), 14-20. doi:10.1016/j.immuni.2014.06.008

Nawaz, A., Aminuddin, A., Kado, T., Takikawa, A., Yamamoto, S., Tsuneyama, K., . . Tobe, K. (2017). CD206(+) M2-like macrophages regulate systemic glucose metabolism by inhibiting proliferation of adipocyte progenitors. Nat Commun, 8(1), 286. doi:10.1038/s41467-017-00231-1

Palevski, D., Levin-Kotler, L. P., Kain, D., Naftali-Shani, N., Landa, N., Ben-Mordechai, T., .. . Leor, J. (2017). Loss of Macrophage Wnt Secretion Improves Remodeling and Function After Myocardial Infarction in Mice. J Am Heart Assoc, 6(1). doi:10.1161/JAHA.116.004387

Parisi, L., Gini, E., Baci, D., Tremolati, M., Fanuli, M., Bassani, B., ... Mortara, L. (2018). Macrophage Polarization in Chronic Inflammatory Diseases: Killers or Builders? J Immunol Res, 2018, 8917804. doi:10.1155/2018/8917804

Pettersson, M., Kelk, P., Belibasakis, G. N., Bylund, D., Molin Thoren, M., \& Johansson, A. (2017). Titanium ions form particles that activate and execute interleukin-1beta release from lipopolysaccharide-primed macrophages. J Periodontal Res, 52(1), 21 32. doi:10.1111/jre.12364

Schneider, C. A., Rasband, W. S., \& Eliceiri, K. W. (2012). NIH Image to ImageJ: 25 years of image analysis. Nat Methods, 9(7), 671-675.

Sica, A., Saccani, A., Bottazzi, B., Polentarutti, N., Vecchi, A., van Damme, J., \& Mantovani, A. (2000). Autocrine production of IL-10 mediates defective IL-12 production and NFkappa B activation in tumor-associated macrophages. J Immunol, 164(2), 762-767.

Tang, Y., Zhao, L., Lei, N., Chen, P., \& Zhang, Y. (2019). Crohn's Disease Patients with Depression Exhibit Alterations in Monocyte/Macrophage Phenotype and Increased Proinflammatory Cytokine Production. Dig Dis, 1-11. doi:10.1159/000501122

Viniegra, A., Goldberg, H., Cil, C., Fine, N., Sheikh, Z., Galli, M., . . Sima, C. (2018). Resolving Macrophages Counter Osteolysis by Anabolic Actions on Bone Cells. J Dent Res, 97(10), 1160-1169. doi:10.1177/0022034518777973 
Wang, X., Li, Y., Feng, Y., Cheng, H., \& Li, D. (2019). Macrophage polarization in aseptic bone resorption around dental implants induced by Ti particles in a murine model. $J$ Periodontal Res, 54(4), 329-338. doi:10.1111/jre.12633

Xue, J., Schmidt, S. V., Sander, J., Draffehn, A., Krebs, W., Quester, I., ... Schultze, J. L. (2014). Transcriptome-based network analysis reveals a spectrum model of human macrophage activation. Immunity, 40(2), 274-288. doi:10.1016/j.immuni.2014.01.006 Yang, J., Zhu, Y., Duan, D., Wang, P., Xin, Y., Bai, L., . . Xu, Y. (2017). Enhanced activity of macrophage M1/M2 phenotypes in periodontitis. Arch Oral Biol. doi:10.1016/j.archoralbio.2017.03.006

Yu, T., Zhao, L., Huang, X., Ma, C., Wang, Y., Zhang, J., \& Xuan, D. (2016). Enhanced Activity of the Macrophage M1/M2 Phenotypes and Phenotypic Switch to M1 in Periodontal Infection. J Periodontol, 87(9), 1092-1102. doi:10.1902/jop.2016.160081

Zhang, C., Yu, X., Gao, L., Zhao, Y., Lai, J., Lu, D., .. . Liu, Z. (2017). Noninvasive Imaging of CD206-Positive M2 Macrophages as an Early Biomarker for Post-Chemotherapy Tumor Relapse and Lymph Node Metastasis. Theranostics, 7(17), 4276-4288. doi:10.7150/thno.20999

Zhang, S., Barros, S. P., Moretti, A. J., Yu, N., Zhou, J., Preisser, J. S., . . Offenbacher, S. (2013). Epigenetic regulation of TNFA expression in periodontal disease. $J$ Periodontol, 84(11), 1606-1616. doi:10.1902/jop.2013.120294

Table 1. Demographic data

\begin{tabular}{|l|c|c|}
\hline & Peri-implantitis lesions & Periodontitis lesions \\
\hline Subjects $(\boldsymbol{n})$ & 7 & 7 \\
\hline Biopsies & 7 & 7 \\
\hline Males: $\boldsymbol{n}$ and (\%) & $3(42.86)$ & $4(57.14)$ \\
\hline Mean Age (years) & $62.0 \pm 12.0$ (Range: $50-80)$ & $58.86 \pm 5.88$ (Range: $30-74)$ \\
\hline
\end{tabular}

Figure legend:

This article is protected by copyright. All rights reserved 


\section{Figure 1: Identification of macrophages in peri-implantitis-associated soft tissue lesions.}

Photomicrographs of macrophages stained for CD68, iNOS, CD206, and counterstained for DNA content using Hoechst 33342 dye. (A) A representative example of peri-implantitis soft tissue depicting co-expression of CD68 and iNOS (M1 polarization), and (B) macrophages co-expressing the CD68 and CD206 marker depicting an M2 polarization. Merged images of all 4 channels depicting enhanced M1 polarization (C) and M2 polarization (D).

\section{Figure 2: Quantification of macrophages from peri-implantitis lesions undergoing M1} and M2 polarization. (A) Quantification of positive macrophages for M1 and M2 polarization demonstrate similar levels of iNOS $^{+}$cells $(53.74 \pm 5.98 \%)$ and $\mathrm{CD}^{206}{ }^{+}$cells $(56.34 \pm 6.91 \%)$ after baseline correction for a total number of $\mathrm{CD}^{+} 8^{+}$macrophages. (B) Percentage of macrophage undergoing M1 and M2 polarization distributed by patient samples ( $n=7$ peri-implantitis patients). Note high heterogeneity on M1 and M2 polarization throughout the patient cohort. (C) The graphic depicts the number of macrophages present within the connective tissue (CT). The results showed the percentage of macrophages found within the total number of cells present in each ROI ( $\mathrm{n}=51$ fields). Note that $14.92 \pm 2.19 \%$ were $\mathrm{CD}^{+} 8^{+}$macrophages, $7.35 \pm 1.44 \%$ were macrophages undergoing M1 polarization, and $7.56 \pm 1.43 \%$ displayed M2 polarization. Data are shown as percentage mean \pm SEM (error bar). Statistical differences are represented as $* *(p<0.01)$.

\section{Figure 3: Macrophage content associated with peri-implantitis and periodontitis lesions.}

(A) Total number of macrophages per field found in peri-implantitis and in periodontitis lesions. Note higher levels of $\mathrm{CD}^{+} 8^{+}$cells in lesions from peri-implantitis $(14.92 \pm 2.19 \%$ positive cells/field) compared to periodontitis lesions $(8.01 \pm 0.32 \%$ positive cells/field) (mean, SEM; ** $\mathrm{p}<0.01$ ). (B) Total number of macrophages undergoing polarization in periimplantitis and periodontitis samples. Note higher levels of M1 macrophage polarization found in peri-implantitis samples $(7.06 \pm 1.44 \%$ positive cells/field, $n=51)$ compared to periodontitis samples $(1.64 \pm 0.14 \%$ positive cells/field, $n=70)$ (mean, $S E M$; $* * p<0.01)$. M2 macrophage polarization presented similar levels on peri-implantitis $(7.56 \pm 1.44 \%)$ and periodontitis $(4.07 \pm 0.20 \%$ ) samples ( $\mathrm{ns}>0.05)$. (C) Macrophages presenting M1 polarization in peri-implantitis samples $(49.93 \pm 5.1 \%)$ compared with periodontitis $(21.04 \pm 2.3 \%)$ after baseline correction for the total number of CD68+ cells $(n=7)$. Data are shown as percentage mean \pm SEM (error bar). Statistical differences are represented as $* * *(p<0.001)$. 

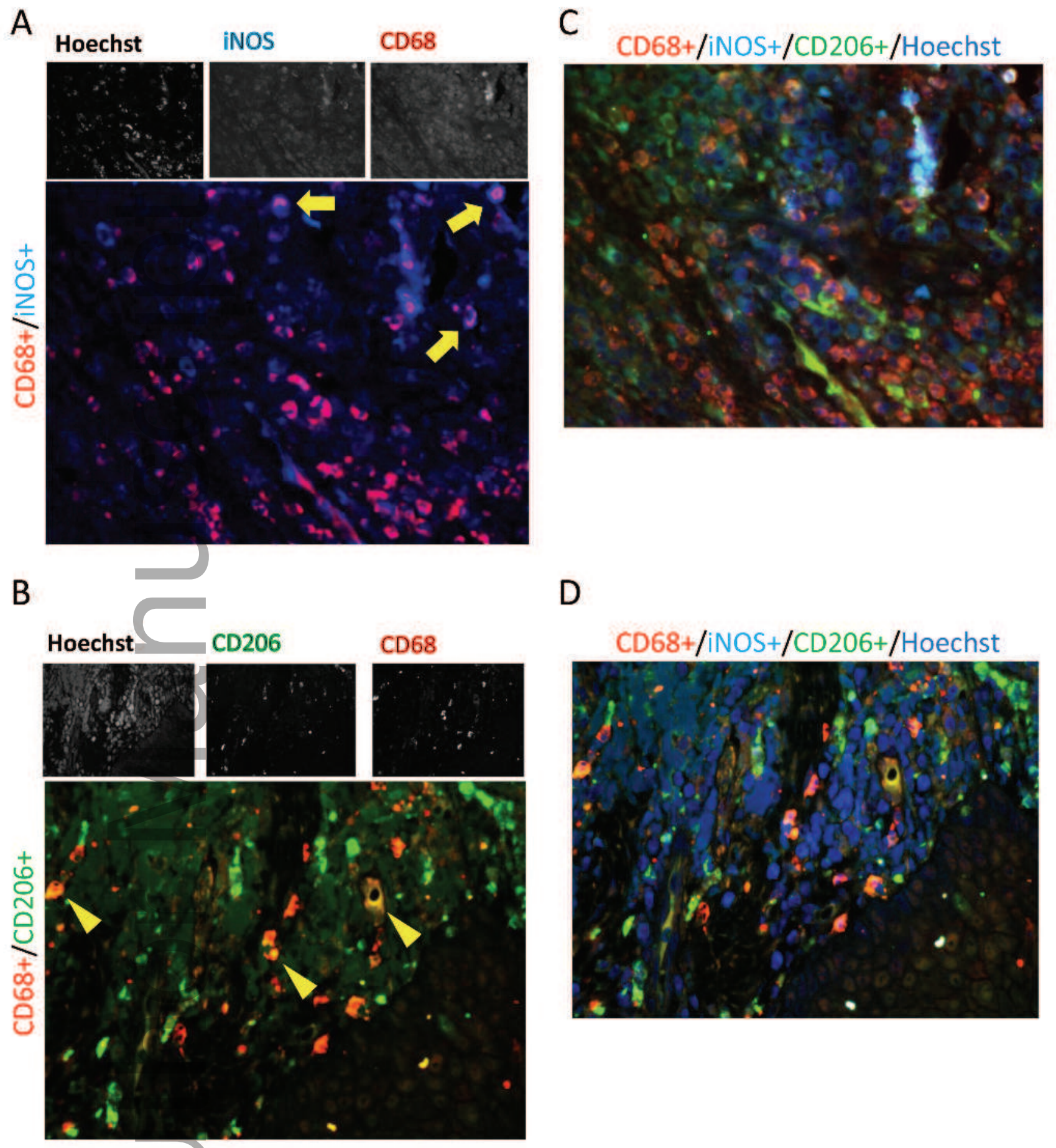

Figure 1: Identification of macrophages in peri-implantitis-associated soft tissue lesions. Photomicrographs of macrophages stained for CD68, iNOS, CD206, and counterstained for DNA content using Hoechst 33342 dye. (A) A representative example of peri-implantitis soft tissue depicting co-expression of CD68 and iNOS (M1 polarization), and (B) macrophages co-expressing the CD68 and CD206 marker depicting an M2 polarization. Merged images of all 4 channels depicting enhanced M1 polarization (C) and M2 polarization (D).

This article is protected by copyright. All rights reserved 

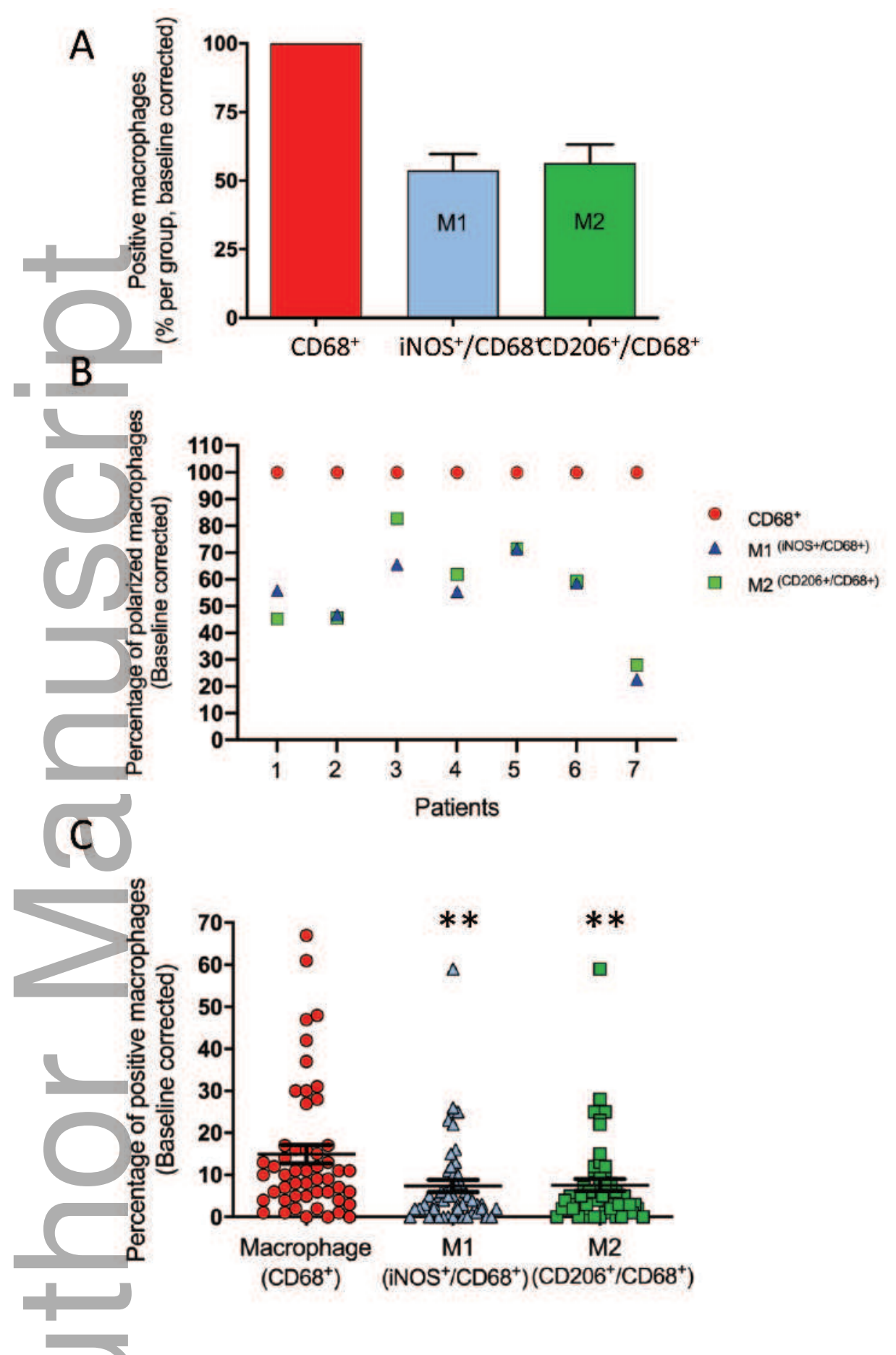

Figure 2: Quantification of macrophages from peri-implantitis lesions undergoing M1 and M2 polarization. (A) Quantification of positive macrophages for M1 and M2 polarization demonstrate similar levels of iNOS+ cells $(53.74 \pm 5.98 \%)$ and CD206+ cells $(56.34 \pm 6.91 \%)$ after baseline correction for a total number of $\mathrm{CD} 68+$ macrophages. (B) Percentage of macrophage undergoing $\mathrm{M} 1$ and $\mathrm{M} 2$ polarization distributed by patient samples $(n=7$ peri-implantitis patients). Note high heterogeneity on M1 and M2 polarization throughout the patient cohort. (C) The graphic depicts the number of macrophages present within the connective tissue (CT). The results showed the percentage of macrophages found within the total number of cells present in each ROI ( $n=51$ fields). Note that $14.92 \pm 2.19 \%$ were CD68+ macrophages, $7.35 \pm 1.44 \%$ were macrophages undergoing M1 polarization, and $7.56 \pm 1.43 \%$ displayed M2 polarization. Data are shown assipercentage mean \pm SEM (error bar hit Statistical differences are represented as ** ( $p<0.01)$. 


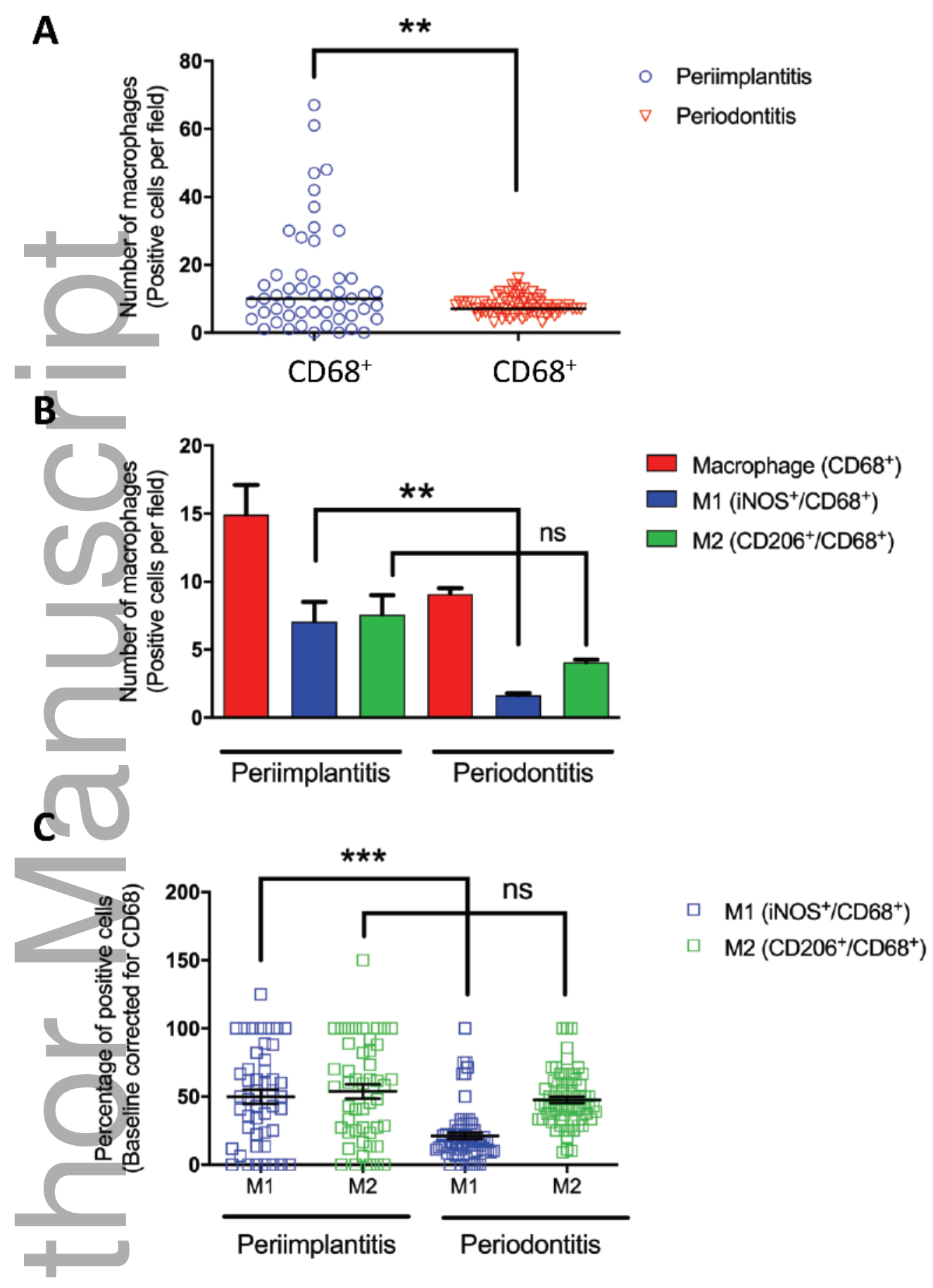

Figure 3: Macrophage content associated with peri-implantitis and periodontitis lesions. (A) Total number of macrophages per field found in peri-implantitis and in periodontitis lesions. Note higher levels of CD68+ cells in lesions from peri-implantitis $(14.92 \pm 2.19 \%$ positive cells/field) compared to periodontitis lesions $\left(8.01 \pm 0.32 \%\right.$ positive cells/field) (mean, SEM; $\left.{ }^{* *} p<0.01\right)$. (B) Total number of macrophages undergoing polarization in peri-implantitis and periodontitis samples. Note higher levels of M1 macrophage polarization found in peri-implantitis samples $(7.06 \pm 1.44 \%$ positive cells/field, $n=51)$ compared to periodontitis samples $(1.64 \pm 0.14 \%$ positive cells/field, $n=70)$ (mean, SEM; ** $p<0.01$ ). M2 macrophage polarization presented similar levels on peri-implantitis $(7.56 \pm 1.44 \%)$ and periodontitis $(4.07 \pm 0.20 \%$ ) samples ( $\mathrm{ns}>0.05$ ). (C) Macrophages presenting M1 polarization in peri-implantitis samples $(49.93 \pm 5.1 \%)$ compared with periodontitis $(21.04 \pm 2.3 \%)$ after baseline correction for the total number of CD68+ cells $(n=7)$. Data are shown as percentage mean \pm SEM (error bar). Statistical

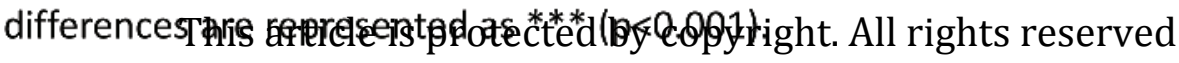

